\title{
Doppler imaging of Speedy Mic using the VLT
}

\section{Fast spot evolution on a young K-dwarf star}

\author{
U. Wolter ${ }^{1}$, J. H. M. M. Schmitt ${ }^{1}$, and F. van $\mathrm{Wyk}^{2}$ \\ 1 Hamburger Sternwarte, Gojenbergsweg 112, 21029 Hamburg, Germany \\ e-mail: uwolter@hs.uni-hamburg.de, jschmitt@hs.uni-hamburg.de \\ 2 South African Astronomical Observatory, PO Box 9, Observatory 7935, South Africa \\ e-mail: fvw@saao.ac.za
}

Received 22 October 2004 / Accepted 15 December 2004

\begin{abstract}
We study the short-term evolution of starspots on the ultrafast-rotating star HD 197890 ("Speedy Mic" = BO Mic, $\mathrm{K} 0-2 \mathrm{~V}, P_{\text {rot }}=0.380 \mathrm{~d}$ ) based on two Doppler images taken about 13 stellar rotations apart. Each image is based on spectra densely sampling a single stellar rotation. The images were reconstructed by our Doppler imaging code CLDI (Clean-like Doppler imaging) from line profiles extracted by spectrum deconvolution. Our Doppler images constructed from two independent wavelength ranges agree well on scales down to $10^{\circ}$ on the stellar surface. In conjunction with nearly parallel $V$-band photometry our observations reveal a significant evolution of the spot pattern during as little as two stellar rotations. We suggest that such a fast spot evolution demands care when constructing Doppler images of highly active stars based on spectral time series extending over several stellar rotations. The fast intrinsic spot evolution on BO Mic impedes the determination of a surface differential rotation; in agreement with earlier results by other authors we determine an upper limit of $|\alpha|<0.004 \pm 0.002$.
\end{abstract}

Key words. stars: activity - starspots - stars: late-type - stars: imaging - stars: individual: BO Mic

\section{Introduction}

Doppler imaging allows the reconstruction of surface maps of a sufficiently fast rotating star. To this end Doppler imaging makes use of the deformations passing through the spectral line profiles as the star rotates. The resolution of these maps depends on the spectral resolution, phase sampling and noise level of the spectra used as input. By making use of information modulated into the line profiles, Doppler imaging overcomes the diffraction limitations of direct and interferometric imaging techniques.

Time series of Doppler images of the same object yield information on the evolution of surface features. This evolution is usually split up into "intrinsic" spot evolution (spot appearance and decay) and large-scale spot motion (tentatively related to meridional flows and differential rotation). Due to the considerable observational effort required for constructing a high resolution Doppler image, in most cases only two images are available of the same object for direct comparison. As a result, the different spot evolution processes can usually not easily be disentangled.

As to intrinsic spot evolution, observational results on spot lifetimes are so far of a rather singular nature, concentrating on a few well-observed objects (Hussain 2002); short-term studies, covering a few stellar rotations are rare. As an example, for the intensively studied apparently single star AB Dor (K0V, $P_{\text {rot }}=0.51 \mathrm{~d}$ ) only the polar spot appears to survive time spans of years (e.g. Donati et al. 1999). We estimate other spots or spot groups, extending several dozen degrees on the surface, to persist longer than about 5 days (Donati \& Collier Cameron 1997a; Donati et al. 1999, as deduced from comparing their Doppler images of AB Dor). Spots of approximately $10^{\circ}$ size (close to the resolution of the available Doppler images) seemingly emerge and decay on similar timescales, i.e. over a few days. However, as e.g. Donati et al. (1999) emphasize, the phase coverage of the compared Doppler images must be checked carefully in order to derive significant lifetime estimates of individual features. For the case of the rapidly rotating G dwarf star He $699\left(P_{\text {rot }}=0.49\right.$ d) the available Doppler images (Barnes et al. 1998) show that the large-scale distribution of spots or spot groups remains stable over a month, while features of approximately $10^{\circ}$ size apparently evolve beyond recognition.

Concerning large-scale surface flows, the presence of slow meridional flows in the solar convection zone (SCZ) is well established. According to helioseismic measurements, these flows extend at least several Mm deep into the SCZ (Gizon et al. 2003). However, long-lived sunspots do not show a regular meridional motion; their meridional displacements 
are apparently dominated by intrinsic evolution (Wöhl 2002). While there are possible indications of meridional movements of the spot pattern for a few evolved stars (e.g. Weber \& Strassmeier 2001), at present no compelling observations of meridional flows exist for solar-like stars other than the Sun.

As to differential rotation, the observational situation is more encouraging. However, determining the rotation law of sunspots (i.e. their differential rotation) requires extended time series of observations. Due to the intrinsic evolution of sunspots it is impossible to derive their rotation law from two solar images alone. On the other hand, for suitable stars other than the Sun this appears to be possible due to the much larger number of spot features available in each surface image. The most prominent example is again AB Dor for which Donati \& Collier Cameron (1997a) measured its differential rotation strength, later confirmed by additional observations (Donati et al. 1999).

The angular velocity $\Omega$ of sunspot rotation as a function of heliographic latitude $B$ can be approximated by (Beck 1999)

$\Omega_{\text {sunspots }}(B) \approx 2.9 \cdot\left(1-0.19 \sin ^{2} B\right) \mu \mathrm{rad} \mathrm{s}^{-1}$.

The precise coefficients of this rotation law depend on the size and lifetime of the sunspots considered, however, deviations are at the level of a few percent. With the same accuracy, this law also describes the rotation of the solar near-surface plasma.

Presently, a rotation law like Eq. (1) is also tentatively assumed for interpreting observations of stars other than the Sun (e.g. Donati \& Collier Cameron 1997a; Reiners \& Schmitt 2003). However, parameters to describe the "strength" of differential rotation can be defined independently of the underlying rotation law. In the following we use

$\alpha=\frac{\Omega_{\mathrm{eq}}-\Omega_{\mathrm{pole}}}{\Omega_{\mathrm{eq}}}$.

For the Sun, this definition results in a value of $\alpha_{\odot} \approx 0.25$. Whether $\alpha$ is a significant physical parameter to describe the dynamics of the outer convection zone is unclear at present.

\section{The target "Speedy Mic"}

Attention was drawn to BO Mic by Bromage et al. (1992), reporting a large flare observed during the EUV all-sky-survey of ROSAT; in retrospect it turned out to be the largest stellar flare observed during the whole mission. Their optical followup observations yielded a radial velocity of $-6.5 \pm 2.0 \mathrm{~km} \mathrm{~s}^{-1}$ with "no evidence for binarity" and an estimated $v \sin i$ of $120 \pm 20 \mathrm{~km} \mathrm{~s}^{-1}$, earning BO Mic its nickname "Speedy Mic". A further analysis of the same photometric data by Anders et al. (1993) did not result in a well-defined rotation period; this issue was settled by Cutispoto et al. (1997), who determined a value of $0.380 \pm 0.004$ days.

In summary, its short rotation period makes BO Mic a promising target for Doppler imaging studies because a full stellar rotation can be observed during a single observing night, allowing the reconstruction of the complete stellar surface. In this way the influence of intrinsic spot evolution during the observations required for a complete Doppler image can be minimized.
A set of Doppler images, based on observations in July 1998 has been published by Barnes et al. (2001). As the authors state, the rather inhomogeneous SNR and phase coverage of their spectra did not allow detailed statements about the small-scale spot evolution or differential rotation on BO Mic.

\subsection{Stellar parameters}

\subsubsection{Magnitudes and colours}

The unusually fast rotation of BO Mic for an apparently single dwarf star suggests a young evolutionary status. Actually, the photometric colours observed by Cutispoto are not consistent with a main sequence star (Cox 2000). The observations of Cutispoto et al. (1997) yielded a visual magnitude at maximum brightness of

$\overline{m_{V}} \approx 9.32 \pm 0.005$.

Together with the HIPPARCOS distance of $44.5 \pm 3.2 \mathrm{pc}$, implying a distance modulus of $3.24 \pm 0.16 \mathrm{mag}$, this results in an absolute visual magnitude of

$\overline{M_{V}} \approx 6.1 \pm 0.1$.

The colours of BO Mic determined by Cutispoto et al. (1997) at brightness maximum are $B-V=0.92 \pm 0.005$ and $V-R=0.56 \pm 0.005$.

\subsubsection{Evolutionary status}

Using the evolutionary models of Siess et al. (2000), including their colour-calibrations, the above values of $M_{V}, B-V$ and $V-R$ can be put into a consistent picture of the evolutionary status of BO Mic. Using models of $0.9 \pm 0.05$ solar masses we find

$\begin{array}{ll}\text { Age } & 3.3 \pm 0.5 \times 10^{7} \mathrm{yr} \\ \text { Radius } & 0.9 \pm 0.05 R_{\odot} \\ T_{\text {eff }} & 4750 \pm 50 \mathrm{~K} .\end{array}$

With $T_{\text {eff }}$ denoting the effective temperature. The errors are determined from the evolutionary models based on the photometric errors given above. They should be considered as rough estimates because of uncertainties of the models and the fact that the maximum brightness state observed by Cutispoto et al. (1997) does presumably not represent a completely spotless hemisphere of BO Mic.

The above radius estimate of $0.9 R_{\odot}$ for $\mathrm{BO}$ Mic resulting from the Siess et al. (2000) evolutionary models is roughly consistent with an estimate based on the observed rotation of BO Mic. An inclination angle of $70 \pm 10^{\circ}$ and a $v \sin i$ of $134 \pm 10 \mathrm{~km} \mathrm{~s}^{-1}$, both discussed in Sect. 5 , result in an equatorial rotation velocity of $v_{\mathrm{eq}}=143_{-17}^{+23} \mathrm{~km} \mathrm{~s}^{-1}$. This further corresponds to a radius of $R=1.07_{-0.14}^{+0.19} R_{\odot}$, using the rotation period of $0.380 \pm 0.004$ days. In light of this, an inclination at the upper limit of the given inclination range $\left(i=60-80^{\circ}\right)$ and a projected rotational velocity at the lower end of 124-144 $\mathrm{km} \mathrm{s}^{-1}$ seem the be the most likely parameters. 


\subsubsection{Lithium abbundance and kinematics}

The $\mathrm{Li} \lambda 6708$ equivalent width of $220 \pm 50 \mathrm{~m} \AA$ determined by Bromage et al. (1992) translates into a $\mathrm{Li}$ abundance of $2.3 \pm 0.4 \mathrm{dex}$, assuming $T_{\text {eff }}=4800 \mathrm{~K}$. (Soderblom et al. 1993c). Because of the large scatter of Li-equivalent widths and abundances for individual stars, this does not allow to closely constrain the age of BO Mic. As an example, the Li $\lambda 6708$ equivalent width ranges from about 100 to $1000 \mathrm{~m} \AA$ at an effective temperature of $4800 \mathrm{~K}$ for the Plejades cluster, age $\approx 70$ Myr (Soderblom et al. 1993c). For members of M 34 (age $\approx 200 \mathrm{Myr}$ ) an abundance of $2.3 \mathrm{dex}$ is approximately the upper limit detected at this effective temperature while for members of the Hyades cluster (age $\approx 600 \mathrm{Myr}$ ) no lithium is significantly detected at effective temperatures below about $5400 \mathrm{~K}$. In summary, the Li $\lambda 6708$ equivalent width of BO Mic indicates an age below "a few" $100 \mathrm{Myr}$ which is compatible with the above value of $30 \mathrm{Myr}$, but does not help to constrain it any further.

Montes et al. (2001) analyze the space velocity of a large sample of stars to check their membership of young stellar kinematic groups; their results concerning the membership of BO Mic in the Local Association (Pleiades moving group) are inconclusive, yielding no further hint of its age.

\section{Observations and data reduction}

\subsection{Spectroscopy}

The spectral observations were performed at the VLT at the ESO Paranal using the spectrograph UVES (Ultraviolet and visual Echelle spectrograph). UVES was used in a dichroic mode, the spectral ranges thus covered were $3260 \AA$ to $4450 \AA$ and $4760 \AA$ to $6840 \AA$ for the blue and red arm, respectively. This permitted the observation of the ranges above $6000 \AA$, well suited for Doppler imaging, and e.g. of the $\mathrm{Ca}$ II $\mathrm{H}$ and $\mathrm{K}$ lines, useful for diagnostics of chromospheric activity. We have not used wavelength ranges below $6000 \AA$ for the Doppler images presented here: the massively increased density of spectral lines for a K-star leads to severe line blending due to rotational broadening for an ultrafast rotator like BO Mic.

The spectrograph slit width was chosen to correspond to 1 " on the sky, offering a spectral resolution of $\lambda / \Delta \lambda \approx 40000$, adequate for the intended Doppler imaging.

BO Mic was observed continuously from dusk to dawn during the two VLT nights (2002 August 2: JD 2452488.53 to JD 2452488.93 , August 7: JD 2452493.51 to JD 2452493.92 ) with the exception of a few reference star exposures. Only the spectra of Gl 472 were used as a template in conjunction with the presented Doppler images.

The exposure times of $\mathrm{BO}$ Mic were adjusted between $140 \mathrm{~s}$ and $200 \mathrm{~s}$ depending on the observing conditions to avoid overexposing the CCDs. The raw exposures were summed in pairs to obtain the spectra finally used for the Doppler imaging. The resulting SNR for both nights ranged typically between 300 and 400 after rebinning to a binwidth of $0.08 \AA$.

The reduction of the spectra was carried out in the IRAF environment. After pre-processing the CCD images and removing scattered light, the echelle orders were extracted by optimum extraction (Horne 1986). Optimum extraction is primarily intended for the extraction of "noisy" spectra, this would not be necessary in this case (even for the poorest spectra the SNR exceeded 200 after rebinning). However, its adaptive modelling of the order extraction apertures could deal efficiently with the spectrum variations induced by the significantly varying seeing conditions (between $0.8^{\prime \prime}$ and $\left.2.5^{\prime \prime}\right)$.

One of UVES' CCDs (the MIT-CCD of the "red arm") shows strong variations (up to about $15 \%$ ) in sensitivity on scales of a few pixels; these variations are visible on technical flatfields as a "brickwall pattern". In order to adequately correct for these small-scale sensitivity variations it was crucial to individually extract a flatfield for each spectrum using its own extraction aperture.

\subsection{Photometry}

The photometric observations were carried out by FvW using the $0.5 \mathrm{~m}$-telescope and modular photometer at the Sutherland site of SAAO (Kilkenny et al. 1988). The standard SAAO reduction procedures were followed, described in the appendix to Kilkenny et al. (1998).

BO Mic was observed continuously during 6 nights (2002 August 3-5, September 12, 13, 15), as far as weather conditions allowed. Unfortunately, the August 2 and August 7 nights were lost due to bad weather at SAAO which inhibited almost simultaneous photometry with our spectral time series. Two E-region standards (Menzies et al. 1989), HD 192844 and HD 193132, were used as comparison star and check star, respectively. The estimated error of the differential photometry is 0.005 mag.

The photometry did not reveal any flares during the observed time span. The September 12-15 lightcurve exhibits the same maxima and minima within $\pm 0.01 \mathrm{mag}$ as its August 3-5 counterpart (Fig. 11). Due to the long time interval between the August and September photometry, corresponding to about 95 rotations of BO Mic, the latter did not add any new information related to the rotation period or the presented Doppler images. As a result, the September photometry of BO Mic is not further discussed in the following.

\section{Line profile extraction using SLSD}

Deconvolving spectra with the aim of extracting "average line profiles" for Doppler imaging was introduced by Donati et al. (1997). Originally this was done in the context of magnetic Doppler imaging to allow the detection of the extremely weak signatures of polarization components. To this end Donati et al. (1997) apply a deconvolution to wide spectral ranges (e.g. spanning more than $1000 \AA$ ) with the aim of massively increasing the SNR of the extracted line profiles compared to individual profiles from the input spectrum.

Donati et al. coined the name "least-squares deconvolution (LSD)" for their implementation of this idea. We call our variant of the same idea "selective least-squares deconvolution (sLSD)". In contrast to the applications of Donati et al., sLSD 
concentrates on the analysis of comparatively narrow wavelength ranges, typically several $10 \AA$ wide, containing only a handful of "strong" lines. While this comes at the price of requiring a higher SNR of the input spectra, SLSD allows to take the local characteristics of the used spectral range into account.

\subsection{The method}

The spectral line profiles of a sufficiently fast rotating star can be approximated by convolving the narrow-lined spectrum of a slowly rotating star (called template spectrum) with a suitable broadening function. The broadening function describes profile shaping mechanisms not confined to small regions of the stellar surface: rotation, limb darkening, macroturbulence and spots. Using this terminology, sLSD tries to approximate a broadening function common to the spectral lines of the selected wavelength region.

The description of LSD by Donati et al. (1997) focusses on a matrix, i.e. a linear formulation of the deconvolution problem. Presently sLSD does not make use of the linearity of the problem, but uses a Levenberg-Marquardt algorithm instead (Press et al. 1992). As in the case of Donati et al.'s LSD, the broadening functions of sLSD are discretely sampled and the value of each sampling bin is treated as one parameter to be optimized. It may appear as a disadvantage of sLSD's not-explicitly-linear treatment that it does not allow a simple error propagation and does not yield a formal error estimate as a byproduct, in contrast to a linear treatment (e.g. Barnes 2004). However, we think that this disadvantage is not very significant, because the errors of the broadening functions, which result from the deconvolution, are clearly dominated by non-statistical errors. This is supported by the results of Barnes (2004) whose "deconvolved profiles" (corresponding to sLSD's broadening functions) show a "rippling effect", most pronounced in the continuum regions surrounding the broadening functions, with an amplitude massively exceeding their estimated statistical errors. Because of this "rippling effect" we have actually excluded these continuum regions around the broadening functions from the deconvolution.

A truly significant error estimate of the broadening functions resulting from sLSD (or LSD) presently appears as an open question. We think that such an error estimate would require a systematic study partly based on synthetic input data, e.g. along the lines of Barnes (2004). However, in addition to Barnes (2004), mismatches of the template spectrum presumably need to be considered: they increase the ill-posedness of the deconvolution problem of SLSD (or LSD), leading to instabilities of the resulting optimization process which could be the reason of the "rippling effect" mentioned above.

A different parametrization of the broadening functions, e.g. by Chebichev polynomials, can be easily implemented in sLSD. However, in practice this does not reduce the number of parameters needed to describe the broadening function: the proper approximation of the large curvature at the transition into the surrounding continuum requires unwieldy high order polynomials.

\subsection{Application and ingredients}

For BO Mic we selected two wavelength regions for the extraction of line profiles by sLSD. They were intentionally chosen as quite different in character (Figs. 1 and 2): While the "6120 2 "region is dominated by a single line (Ca I 16122$)$, the "6400 $\AA$ "region comprises a handful of stronger lines (the strongest are Fe I $\lambda 6400$ and $\mathrm{Ca}$ I 26439 ).

Figures 1 and 2 illustrate the application of sLSD to the two wavelength ranges. In the large panels the gray broadlined curve represents the observed spectrum, the black spectrum shows the fit achieved by SLSD. The vertical dotted lines delimit margin regions contributing to the spectrum fit with reduced weights; the narrow flat regions around $6404 \AA$ and $6434 \AA$ in Fig. 1 have been excluded from the fit (see below in Sect. 4.2.1).

The narrow-lined spectra show the templates used for the deconvolution. In case of Fig. 1 a synthetic spectrum generated for an effective temperature of $5200 \mathrm{~K}$ by the spectrum synthesis code PHOENIX (Hauschildt et al. 1999) has been used. The deconvolutions shown in Fig. 2 make use of an observed template spectrum (G1 472, K1V); spectra synthesized by PHOENIX tentatively adopting a wide range of element abundances and effective temperatures yielded significantly poorer spectral fits in this wavelength region.

The application to relatively narrow wavelength intervals leads to several issues to be treated by SLSD: the treatment of end-effects at the borders of the wavelength interval, tentatively correcting for small template deficiencies and stabilizing the convergence of the broadening function optimization. These issues are discussed below in Sects. 4.2.1 and 4.2.2.

\subsubsection{End-effects and template deficiencies}

Figure 3 shows an example application of sLSD using the same template as in Fig. 1, but yielding a poorer fit to the input spectrum. This rather poor spectral fit is due to mismatches with the template spectrum (leading to slightly wrong equivalent widths of some lines, most pronounced in the region around $6360 \AA$ ) and regions containing echelle order overlaps not optimally merged (the region near $6460 \AA$ ). To avoid the resulting deficiencies of the fit shown in Fig. 1, three measures have been taken: (i) narrowing down the wavelength region, carefully selecting the boundaries of the fitted wavelength range and excluding narrow intervals from the spectral fit (near $6403 \AA$ and $6435 \AA$ ); (ii) defining margin intervals at the boundary of the fitted range where the relative weights used for the $\chi^{2}$-optimization of the spectral fit are reduced; these margin intervals are marked by vertical lines in Figs. 1 and 2; (iii) introducing an adaptive "continuum correction function" (CCF), shown in the lower narrow panels of Figs. 1 to 3.

The exclusion of narrow wavelength intervals from the spectral fit, mentioned in item (i), is motivated by template spectrum mismatches, i.e. apparently missing weak lines in these intervals. These missing lines in the synthetic template spectrum may be due to wrong element abundances supplied to PHOENIX or wrong atomic data. However, we were unable to find element abundances leading to an improved spectral fit, 

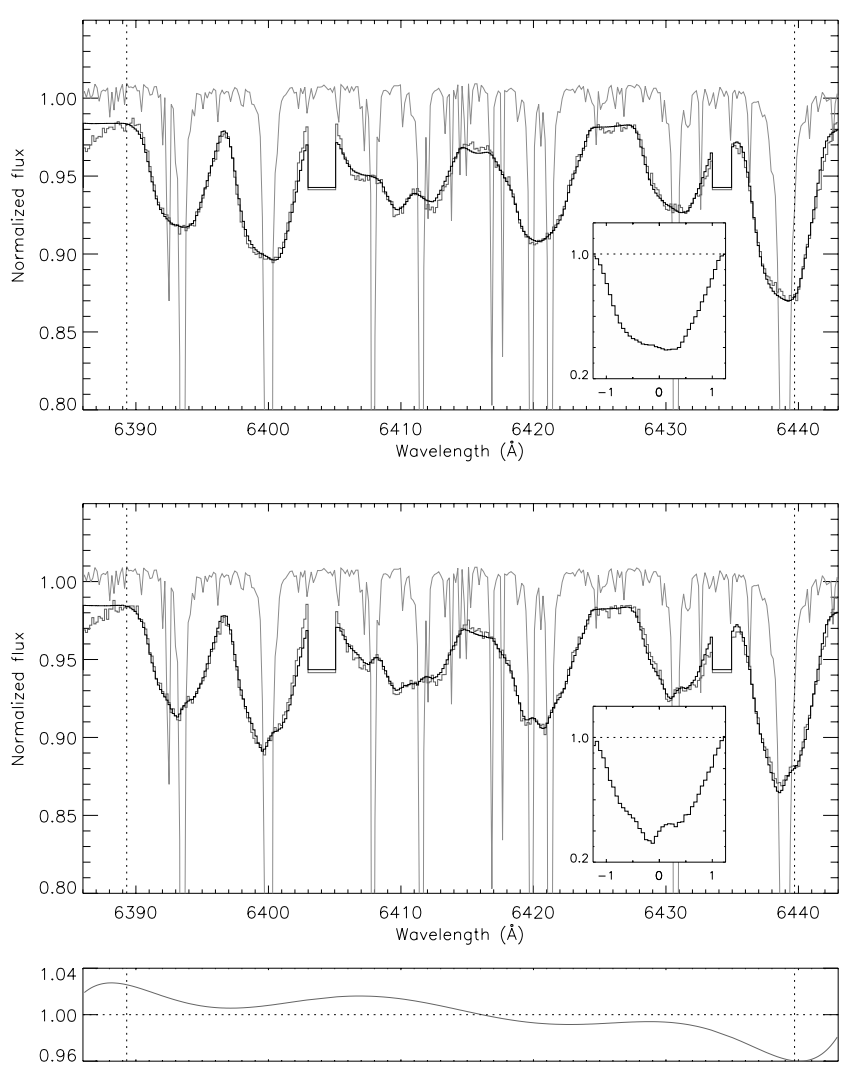

Fig. 1. Application of spectrum deconvolution (sLSD) to the "6400 $\AA$ " wavelength region of two spectra of BO Mic. In the large panels the gray broad-lined curve represents the observed spectrum, the black spectrum shows the fit by sLSD. The narrow-lined spectrum is the template used for deconvolution (a synthetic PHOENIX $5200 \mathrm{~K}$ spectrum without rotational broadening). The inset panels show the resulting line profiles (broadening functions), their $x$-axes are annotated in units of $120 \mathrm{~km} \mathrm{~s}^{-1}$. The lowermost panel shows the "continuum correction function" (CCF) used for both spectra; see text for details.

so wrong atomic data seem more plausible. Actually, the exclusion of the intervals is not necessary in this case, because it has only a marginal influence on the line profiles resulting from the deconvolution.

Measure (ii), namely the margin intervals, would not be necessary if the selected wavelength range were surrounded by practically line-free regions, i.e. intervals of "undisturbed" continuum. However, such undisturbed regions are largely absent in the visible spectra of very fast rotating cool stars (which are the primary candidates for Doppler imaging). By introducing the margin intervals, SLSD reduces the influence of strong lines completely or partly outside the selected wavelength range.

The most important measure to reduce this influence is the application of the above named CCF. The observed spectrum is divided by the CCF prior to the optimization of the broadening function. The CCF is chosen as a slowly varying function of wavelength, i.e. varying on larger scales than the individual line profiles of the spectrum. In this way the CCF adapts the continua of the observed and template spectra. This makes a continuum normalization of the input spectra prior to SLSD unnecessary. Such a normalization is usually very difficult for
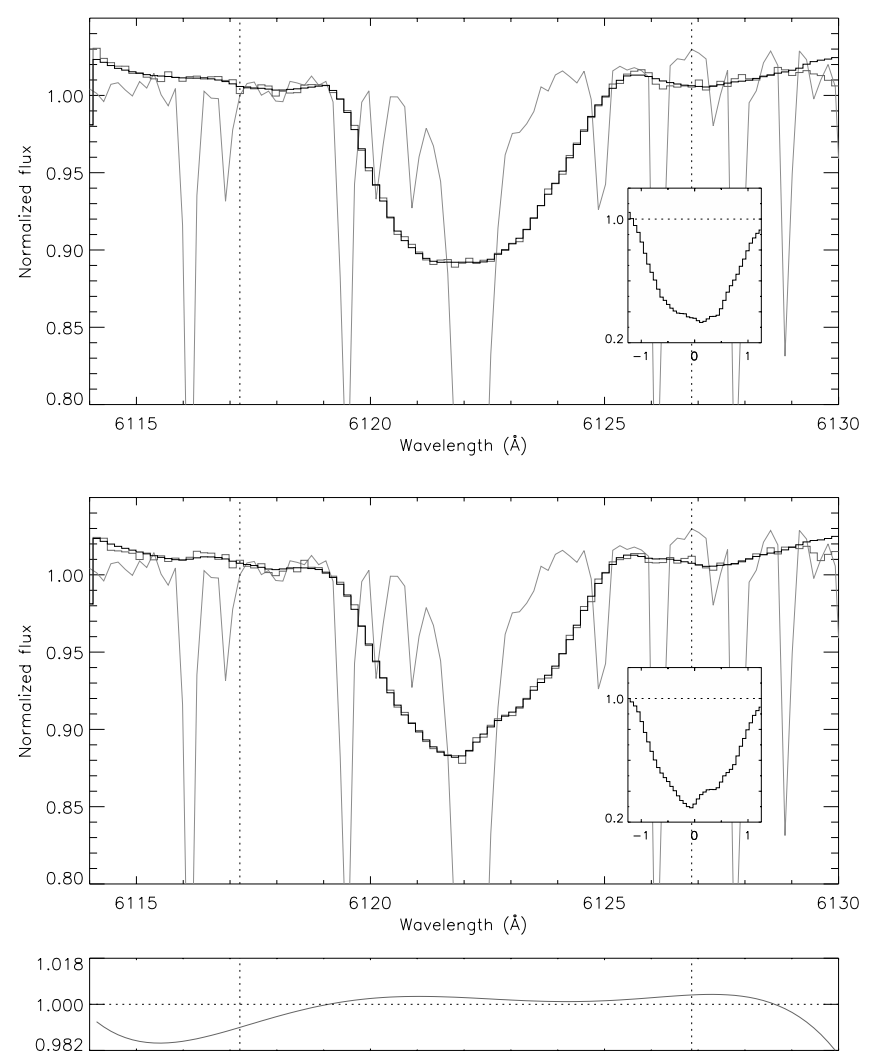

Fig. 2. Application of sLSD to the " $6120 \AA$ " wavelength region; the input spectra are the same as in Fig. 1, observed on 2002 August 2 at rotation phases 0.337 and 0.639 , respectively. For this wavelength range an observed template spectrum (Gl 472) was used.
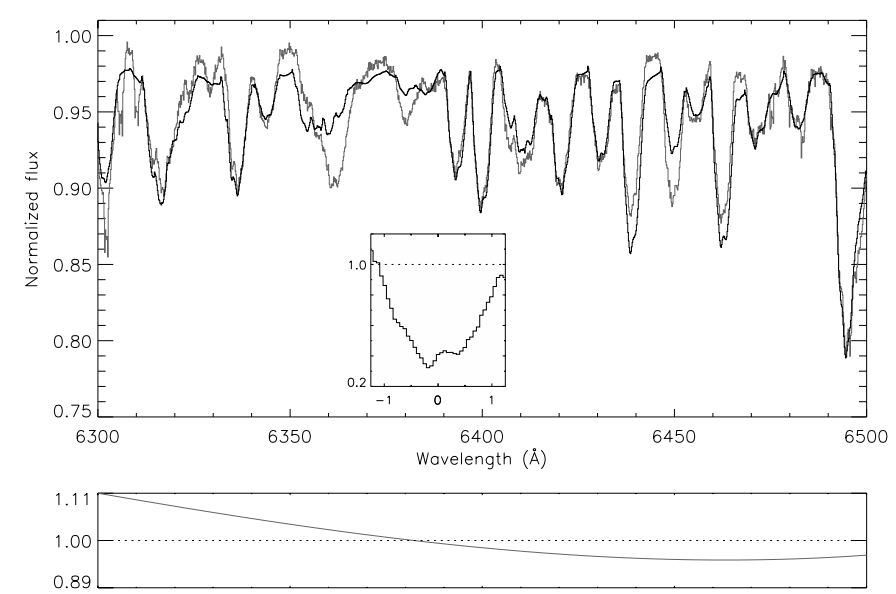

Fig. 3. Application of sLSD to a moderately wide spectral range around the "6400 ^" wavelength region. The input and template spectra are the same as in the lower panel of Fig. 1, the latter is not shown here. The reasons for the rather poor fit to the spectrum are discussed in the text.

strongly rotationally broadened spectra. It remains so when using the $\mathrm{CCF}$, but the process can be monitored during the application of SLSD, instead of being potentially "hidden" in the original spectra reduction. Additionally the CCF compensates for the effects of strong lines at the boundary of the wavelength region. This compensation is clearly visible in Figs. 1 and 2 showing the deformations of the $\mathrm{CCF}$ at the boundaries. 
Finally the CCF tentatively corrects for some deficiencies of the template spectrum (wrong equivalent widths or missing weak lines). An example for such a correction can be seen when comparing the region around $6410 \AA$ in Figs. 1 and 3.

The CCF is iteratively constructed by alternating optimization steps for the broadening function and the CCF. The CCF is chosen as a Legendre polynomial of fixed degree; this degree needs to be chosen sufficiently high to provide "flexibility" for the intended corrections. On the other hand, the degree should be adequately low to avoid the CCF adapting to deformations of individual line profiles which are to be fitted by the broadening function. As an example, the degrees are 7 in Fig. 1, 5 in Fig. 2 and 3 in Fig. 3, respectively.

For the extraction of a time series of line profiles the CCF should be kept fixed, or its constancy closely monitored. Otherwise variations of the CCF, e.g. due to instabilities of the optimization could introduce "fake" variations of the extracted line profiles.

\subsubsection{Stabilizing the convergence}

During the early phase of the broadening function optimization small-scale oscillatory solutions (typically with large bin-tobin amplitudes) tend to appear. This tendency is further enhanced by the alternating optimization of the CCF and the broadening function. These oscillatory solutions massively disturb or even prevent the convergence of SLSD. To avoid this, sLSD employs a Tikhonov-regularization of the optimization of the broadening function; i.e. it applies a "penalty function" to suppress strong small-scale gradients of the solution. The smoothing effect of the Tikhonov-regularization on the solution profile is adjusted by the weight of the penalty function during the optimization. This weight is fixed prior to the sLSD iteration; for input spectra of low or moderate noise it can be chosen very small. In this way it suppresses the oscillatory solutions without noticeably smoothing the final solution profiles, i.e. without a loss of potential starspot signatures in the profiles.

\subsection{Selecting the template spectrum}

The observed stellar spectrum results from a disk integration and is in effect a weighted sum of contributions from undisturbed photospheric regions and spots. As a consequence, (at least) two intrinsic spectra take part in the disk integration, but only one template is used for the deconvolution. Since the disk integration is dominated by the spectrum of the undisturbed photosphere, due to its greater brightness, the template spectrum for the deconvolution needs to be selected similar to it. In this way the intrinsic spectrum (or even different spectra) of the spots is not included as input information to the spectrum deconvolution.

If the intrinsic spectra of the unspotted and spotted photosphere were identical, extracting a broadening function common to the whole stellar surface would be strictly correct. Naturally, because of the strong temperature contrast and other differing atmospheric parameters, these spectra are not identical. However, Doppler imaging based on spectrum
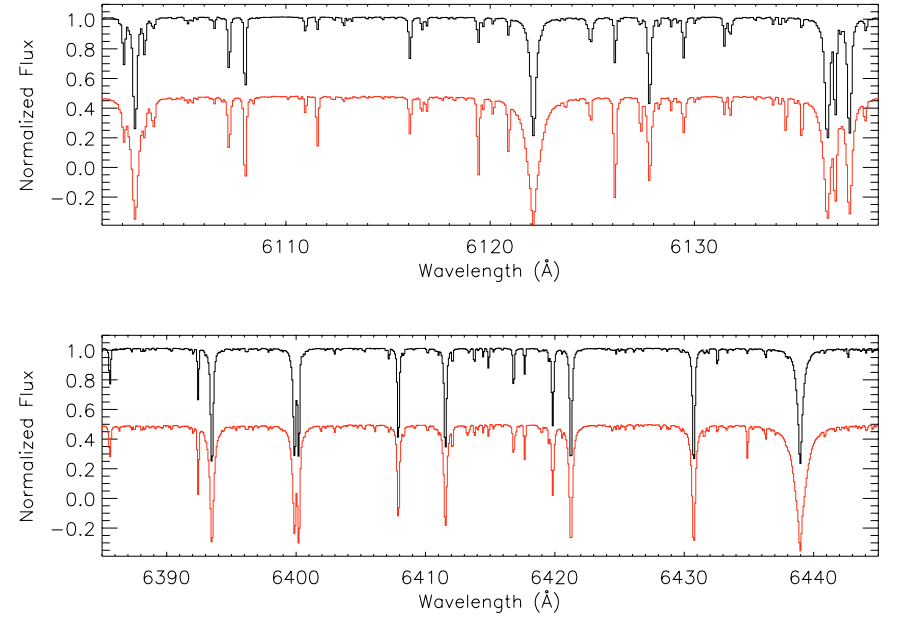

Fig. 4. Selected ranges from synthetic spectra, not rotationally broadened. The PHOENIX $5200 \mathrm{~K}$ spectrum (black) has been used as the template spectrum for applying sLSD to the " $6400 \AA$ ^" wavelength range. The PHOENIX $4600 \mathrm{~K}$ spectrum (red/gray) is considered as a proxy for the spectrum of the spots on BO Mic, see text for details. For both panels, the quasi-continua have been normalized to unity, the red/gray spectrum is shifted in flux by -0.5 .

deconvolution apparently is quite successful as demonstrated by the works of Donati et al. and also supported by the results of this paper. As discussed in the following, such a success can only be expected if the spot-photosphere temperature contrasts are either small or large, in a sense to be defined below.

Figure 4 illustrates a spot-photosphere temperature contrast which is small compared to e.g. the Sun. The spectrum of lower effective temperature is used here as a tentative proxy for the spectrum of the spots on BO Mic, the temperature contrast has been chosen as $600 \mathrm{~K}$ in this example. This approximately represents the situation assumed for the construction of the Doppler images discussed in Sect. 5.3, because in the considered wavelength range the ratio of the quasi-continuum fluxes of the two spectra is about 0.5 .

In this case the dominating lines in the shown wavelength ranges are very similar for the spots and the undisturbed photosphere. As a result, the line profile deformation by cold spots is predominantly due to the spot-induced change of continuum flux and approximately correctly recovered by the deconvolution with a single template spectrum. Obviously, this does not apply to spectral lines strongly varying with temperature; consequently they should be avoided when using SLSD for the line profile extraction.

If on the other hand a large spot-photosphere temperature contrast is assumed (e.g. $1800 \mathrm{~K}$, as deduced for the highly active K-dwarf LQ Hya by Saar et al. 2001), the flux emitted by the spots is very small compared to the surrounding photosphere (less than $10 \%$ in the example of LQ Hya, estimating from PHOENIX synthetic spectra of corresponding effective temperatures). In this case the intrinsic spectrum of the spots has very little influence on the resulting broadening function. Again, the deconvolution based on a single template spectrum should produce approximately correct results. 


\section{Doppler imaging using CLDI}

\subsection{The method}

We call the Doppler imaging algorithm used in the following "CLEANlike Doppler imaging" (CLDI), it is based on the algorithm developed by Kürster (1993). CLDI is described in detail in Wolter (2004), further descriptions and studies of its behaviour will appear in a separate publication (Wolter 2005, in preparation). CLDI's rationale is very similar to the algorithm described and tested in Kürster (1993).

The mathematical formulation of the Doppler imaging problem results in a matrix equation, $D=\boldsymbol{R} \cdot I$ (e.g. Vogt et al. 1987). For this formulation, the line profiles are discretely sampled and assembled end-to-end giving a one-dimensional vector $D$. Likewise, the surface has been divided into a discrete grid of zones whose values have also been packed into a onedimensional vector $I$. The matrix $\boldsymbol{R}$ is the so-called response matrix. At the given resolution it describes the observable line profiles as a function of the stellar surface.

Doppler imaging is an inverse problem making this equation ill-posed and ill-conditioned (e.g. Lucy 1994). In a way, CLDI approaches the equation in a mathematically "daring" way: since the inverse $\boldsymbol{R}^{-1}$ does not exist, it attempts a solution of the above equation by iteratively applying the transpose of the response matrix $\boldsymbol{R}^{\top}$ to the observed line profile deformations. More precisely, it applies a slightly modified $\boldsymbol{R}^{\top}$ to the difference of the observed line profile deformations and their reconstructed counterparts at each iteration step. As discussed in Wolter (2004), this iterative "backprojection" of the line profile deformations onto the reconstruction surface can be justified by a geometric interpretation of $\boldsymbol{R}^{\top}$.

CLDI is intended as an alternative to regularized optimization schemes for the inversion of Doppler imaging problems, like maximum-entropy Doppler imaging. In contrast to methods based on regularization, CLDI does not explicitly treat Doppler imaging as a continuous optimization problem. Instead it attempts a discrete approximation of the observed line profile deformations in the way described above. In this way, inspired by the appearance of sunspots, CLDI models surface reconstructions of high contrast and discrete contrast steps.

\section{2. $s L S D$ and CLDI}

The line profiles (more precisely the broadening functions, cf. Sect. 4.1) extracted from average spectra of BO Mic using sLSD show minor deviations from symmetry, an example is shown in Fig. 5.

Phase-independent line profile asymmetries massively disturb the convergence of CLDI. The reason is that even weak deviations of this kind mislead CLDI's "backprojection" of the input line profiles used for approximately locating (groups of) spots on the surface. Such deviations are intrinsically impossible to fit for Doppler imaging, because they lie outside the line center without migrating through the profile with rotation phase. We therefore have to make sure that the time series of line profiles is as far as possible free of such phase-independent asymmetries.

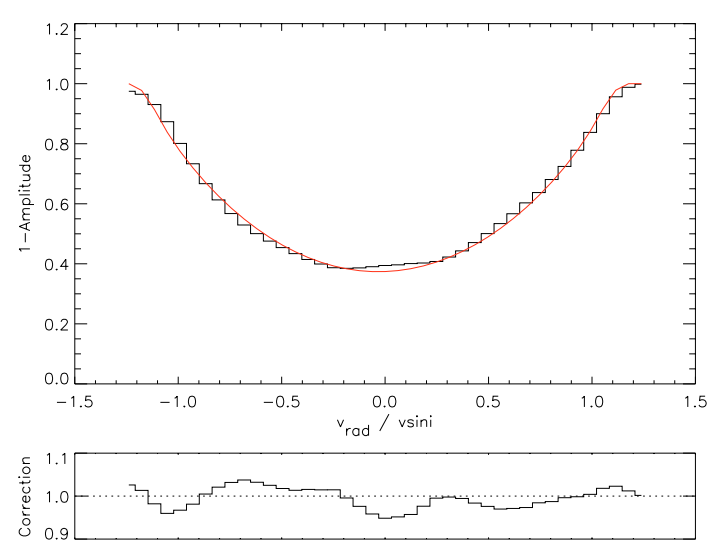

Fig. 5. Upper panel: average line profile of BO Mic (stepped graph) extracted by sLSD from the "6400 $⿱$ ^" wavelength region; the radial velocity is annotated in units of $120 \mathrm{~km} \mathrm{~s}^{-1}$. The smooth graph shows a fitted analytical rotation profile, its parameters are given in Table 1. Lower panel: correction function $\xi$ computed from Eq. (7). See text for details.

Such asymmetries demand care when interpreting the shape of the line profile in terms of stellar parameters (e.g. Reiners \& Schmitt 2003). For the purpose of Doppler imaging these asymmetries are less crucial, if they are small compared to the spot-induced line profile deformations.

In order to ensure the convergence of the Doppler imaging, the line profiles extracted from the spectra of BO Mic have been transformed to symmetric rotation profiles in the following way, illustrated in Fig. 5. The shown profile was extracted from an average of all spectra of BO Mic taken on August2, these spectra sample a whole stellar rotation quite evenly. In this way spot-induced line profile deformations should largely cancel out, assuming that the spot pattern does not change during the observations.

A symmetric rotation profile is determined by fitting an analytic rotation function $f_{\text {rot }}$ to an average line profile of the time series. $f_{\text {rot }}$ is computed from a "rotation profile" $G$ (Gray 1992)

$G\left(v_{\mathrm{rad}}\right)=\frac{2(1-\epsilon) \mathrm{A}^{\frac{1}{2}}+\frac{1}{2} \pi \epsilon \mathrm{A}}{\pi W(1-\epsilon / 3)}$

by convolving it with a Gaussian of constant width. Here the symbol $\mathbf{A} \equiv 1-\left(v_{\text {rad }} /(c \cdot W)\right)^{2}$ has been used, computed from

$W=\frac{v \sin i}{c} \lambda_{0}$

using the center wavelength of the considered line $\lambda_{0}$.

A correction function $\xi$ is computed from the average line profile $b_{\text {ave }}$ and the corresponding analytic fit $f_{\text {rot }}$ :

$\xi\left(v_{\mathrm{rad}}\right)=\frac{f_{\mathrm{rot}}\left(v_{\mathrm{rad}}\right)}{b_{\mathrm{ave}}\left(v_{\mathrm{rad}}\right)}$

A sample correction funtion is shown in the lower panel of Fig. 5 , in that case the amplitude of $\xi$ is about 0.03 , i.e. the correction amounts to at most $3 \%$ of the continum surrounding the line profile. Each individual line profile of the time series is then corrected by applying the same correction function

$b_{\mathrm{corr}}\left(v_{\mathrm{rad}}\right)=b\left(v_{\mathrm{rad}}\right) \cdot \xi\left(v_{\mathrm{rad}}\right)$. 
The thus corrected $b_{\text {corr }}$ are used as the input to CLDI. Using the same correction $\xi$ for the whole time series is a natural requirement; it avoids introducing artificial phase-dependent deformations of the line profiles, which could cause artefacts in the resulting Doppler images.

Care must be taken that symmetric deviations between the average input profile $b_{\text {ave }}$ and $f_{\text {rot }}$ are not "corrected away" during this process. Such symmetric deviations are induced e.g. by polar spots (and other rotationally symmetric spot configurations). As illustrated by Fig. 5, such signatures were not detected for the average profiles of BO Mic.

\subsection{The images}

Two independent time series of line profiles were extracted from the spectra of BO Mic (Sect. 4.2), the corresponding images are denoted by " $6120 \AA$ ” and " $6400 \AA$ " in the following. The images are shown in Fig. 6, the achieved line profile fits are shown for one of them in Fig. 7.

As stated above, the SNR of the input spectra ranged typically between 300 and 400, for some spectra it reached up to 500. Only for 3 of the spectra was the SNR below 250. We have carried out tests of CLDI using synthetic input profiles with very similar reconstruction parameters as those used for our reconstructions of BO Mic (Wolter 2005, in preparation). These tests suggest a "critical” SNR range for the Doppler imaging reconstructions, mainly depending on the resolution aimed at and on the adopted atmospheric parameters. When the SNR of the input profiles falls below this critical range, a serious deterioration of image details results. Above this level, the reconstruction quality only increases weakly with increasing SNR. Our tests indicate that this critical SNR range lies between about 200 and 250 for the parameters adopted here.

\subsubsection{Image reconstruction parameters}

The image reconstruction parameters are summarized in Table 1 . The limb-darkening and $v \sin i$ values have been determined by fitting analytical rotation profiles $f_{\text {rot }}$ to average line profiles (broadening functions) of BO Mic (Sect. 5.2). It should be noted, that the errors of the fit parameters are presumably larger than the margins given in the table. This is mostly due to the asymmetries of the average line profiles, introducing uncertainties into the fit ${ }^{1}$.

The adopted value of 0.5 for the spot continuum flux means that a surface element completely covered with spots (i.e. with a spot filling factor of one) emits half of the flux in the continuum around the synthesized line profile, compared to an uncovered surface element (spot filling factor zero). Adopting a lower value, i.e. a larger spot-photosphere contrast, leads to a slightly more "jagged" appearance of the spot groups of the resulting Doppler images, it has no significant influence on the reconstructed spot pattern.

\footnotetext{
${ }^{1}$ Analyzing the same profiles in Fourier space (Reiners \& Schmitt 2002a) yields $v \sin i \approx 131 \pm 2 \mathrm{~km} \mathrm{~s}^{-1}$. However, since this method is also affected by problems of profile asymmetry, the error may well be larger.
}

Table 1. Parameters adopted for the construction of the Doppler images of BO Mic, $\epsilon$ represents the linear limb darkening coefficient. The spot continuum flux is given relative to an undisturbed photospheric continuum flux of one. Note that the given margins designate the deviations between parameters adopted for different reconstructions, they are not proper error estimates.

\begin{tabular}{|c|c|}
\hline Rotation period & $P_{0}=0.380$ days \\
\hline Inclination & $70^{\circ}$ \\
\hline Spot continuum flux & 0.5 \\
\hline$v \sin i$ & $134 \pm 2 \mathrm{~km} \mathrm{~s}^{-1}$ \\
\hline \multirow[t]{2}{*}{$\epsilon$} & $0.9 \pm 0.1$ (“6120 ̊̊”) \\
\hline & $0.7 \pm 0.1$ (“6400 ̊’) \\
\hline
\end{tabular}

\subsubsection{Inclination}

Adopting an inclination for Doppler imaging is commonly done by attempting reconstructions at different inclinations and selecting the one yielding the best line profile fits. Figure 8 shows the line profile fit quality expressed as $\chi_{\text {final }}^{2}$ as a function of the adopted inclination for different datasets of BO Mic. These curves do not show a strong variation of $\chi_{\text {final }}^{2}$; this behaviour is not typical of CLDI, as we have tested for surfaces reconstructed from synthetic data.

The minima of $\chi_{\text {final }}^{2}$ for the August 7 reconstructions coincide at $70^{\circ}$. The August 2 reconstructions show less pronounced minima at lower values which do no agree. As discussed above, the radius of BO Mic tentatively deduced from evolutionary models clearly suggest an inclination $\gtrsim 70^{\circ}$. As a result this value has been chosen for the reconstructions shown here. Actually varying the inclination by as much as $\pm 20^{\circ}$ has only little influence on the resulting Doppler images.

\subsection{Surface resolution}

The resolution achieved by a Doppler image depends on the phase sampling, the spectral resolution and the noise of the spectra used for its reconstruction. Due to the one-dimensional projection of the surface structures onto each individual line profile, the resolution also depends on the reconstructed latitude. Although analytic estimates exist (e.g. Jankov \& Foing 1992) they are not much in use, because the quantitative influence of the noise on the image resolution depends e.g. on the adopted atmospheric parameters and requires simulations based on synthetic input data. In addition, potential errors of the line profile modelling, imperfectly treated line blends etc. can severely deteriorate the reconstruction quality. An extensive series of such simulations can be found in Rice \& Strassmeier (2001). We have studied the behaviour of CLDI based on synthetic input data (Wolter 2004; 2005, in preparation). It has already been extensively studied for CLDI's predecessor in Kürster (1993) with essentially the same results as for maximum entropy Doppler imaging.

For the purpose of this paper, the resolution estimate is based on a comparison of the two presented sets of images. They are reconstructed from two disconnected spectral regions of rather different character (concerning the number of lines 

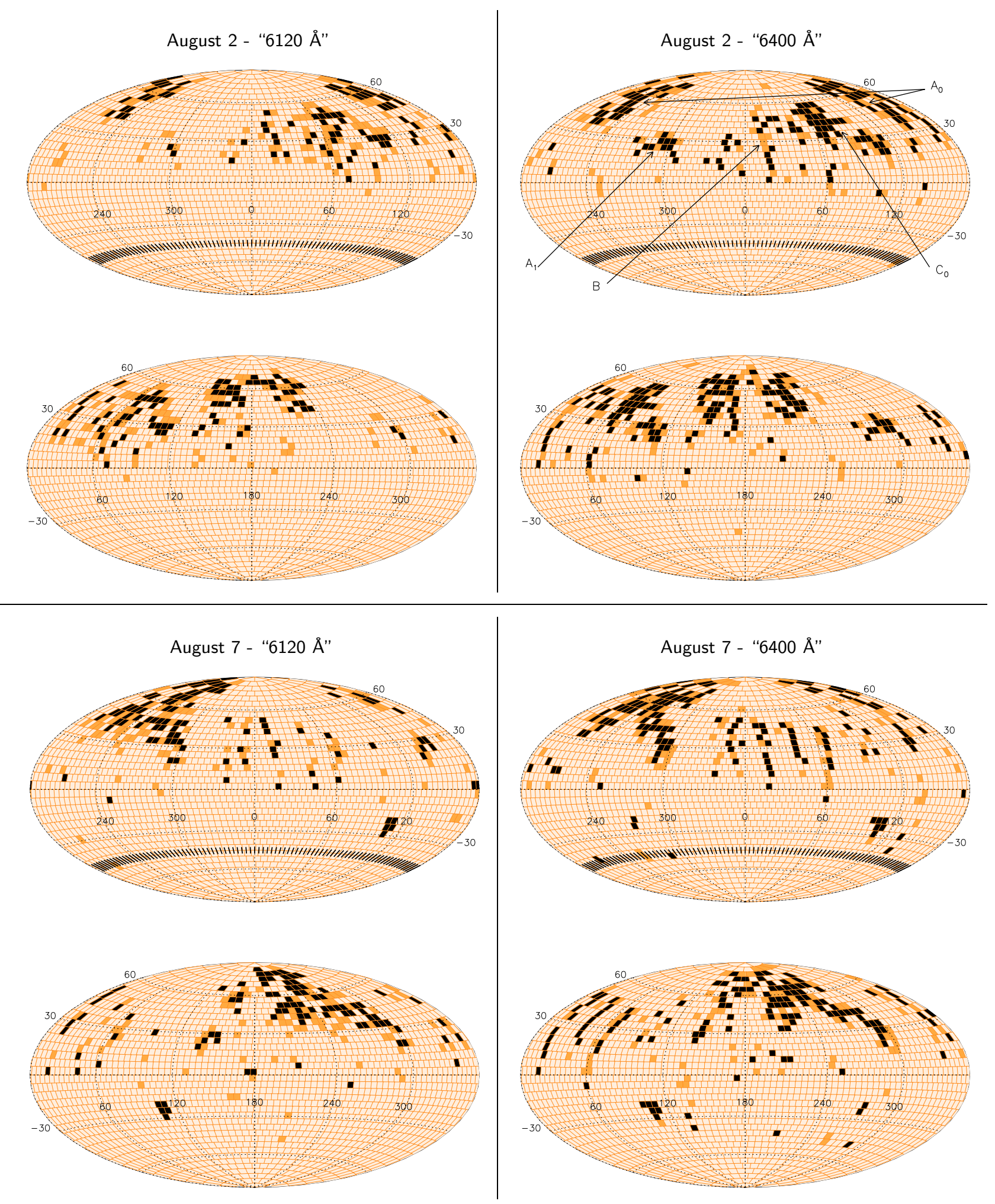

Fig. 6. Doppler images of BO Mic, reconstructed by CLDI from the wavelength region given above each panel. The lower and upper map of each panel show the same surface, but rotated by 180 degrees. Black and dark gray/orange areas represent surface regions completely covered or $50 \%$ covered with spots, respectively. Unspotted regions are rendered as light shades. The subobserver longitudes of the observed phases are marked by short lines. A rotation phase $\phi=0$ corresponds to a subobserver longitude of $\varphi=0^{\circ}$; rotation proceeds with decreasing subobserver longitude. About 13 stellar rotations have passed between the images of the upper and the lower panels. 

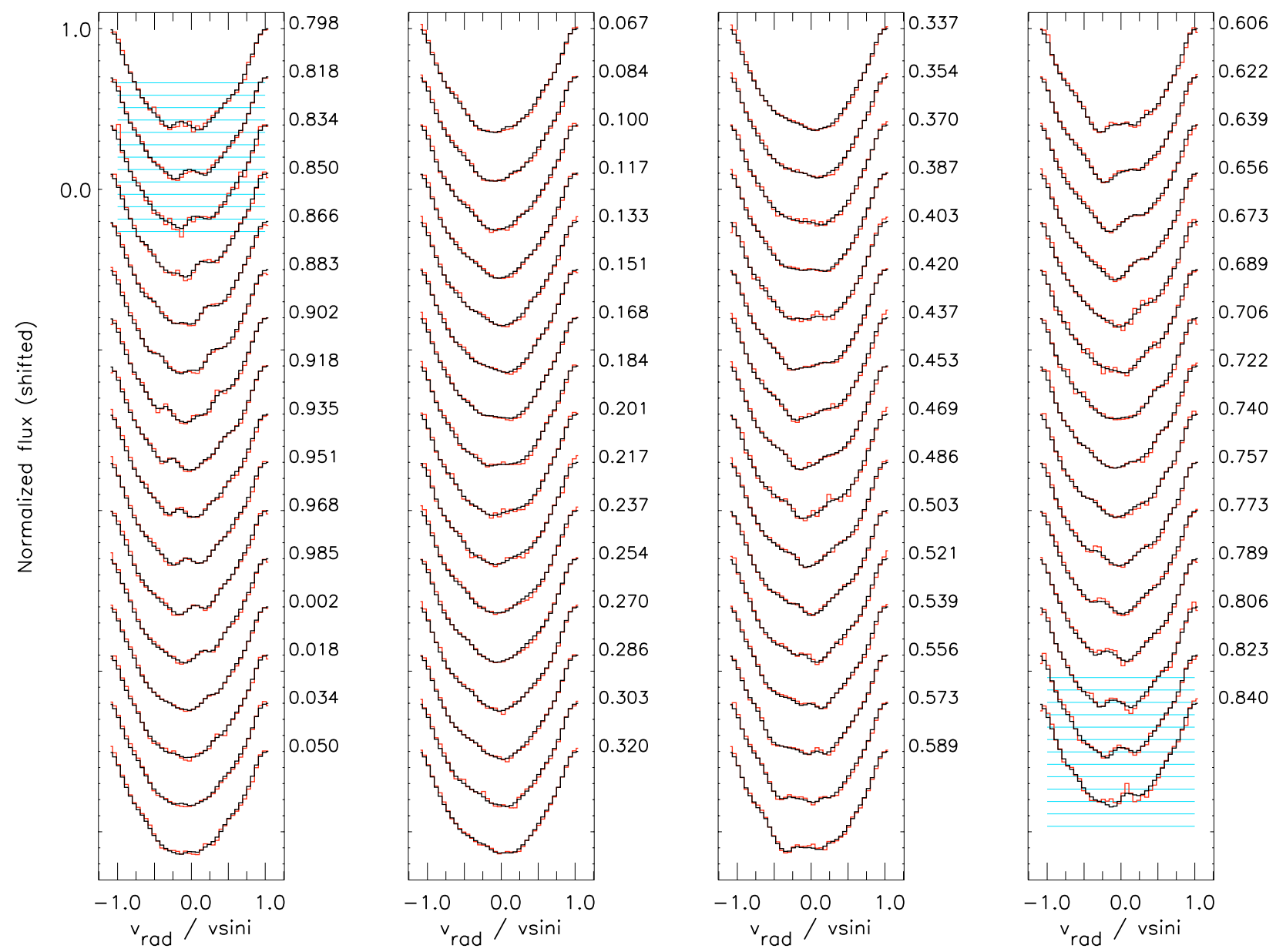

Fig. 7. Line profiles of BO Mic, extracted by spectrum deconvolution in the "6400 ^"-region (red/gray) and fitted by CLDI (black). The reconstruction parameters are listed in Table 1. Subsequent profiles of the time series are shifted, the flux scale is valid for the topmost profile of each panel. The rotation phases are given right of each profile, with the phase zero-point at $\mathrm{JD}_{\phi=0}=2448000.05$ Both observation nights were longer than the rotation period of 0.380 days, the hatched regions mark the cores of the profiles in the phase overlap interval of the beginning and the end of the night.

and the degree of line blending they contain). As a result, they can be considered as quite independent input data describing the same surface patterns.

The images of Fig. 6 belonging to the same input spectra (i.e. to the same dates) show corresponding features on scales down to about $10^{\circ} \times 10^{\circ}$ on the surface; the correspondence becomes poorer within about $15^{\circ}$ from the pole and the equator. This somewhat subjective estimate is substantiated by the surface cross-correlation (e.g. Tonry \& Davis 1979) shown in Fig. 9: the correlation peaks are located within $\pm 10^{\circ}$ in the given latitude range. The cross-correlation was computed along longitude for each latitude strip of the compared Doppler maps.

\section{Results}

\subsection{Observed spot evolution}

Comparing the August 2 and August 7 images, several spot groups are recognizable (Fig. 6):

$A_{0}$ : a large irregularly shaped spotted area extending from about $120^{\circ}$ to $240^{\circ}$ in longitude and from about $30^{\circ}$ up to above $60^{\circ}$ in latitude;
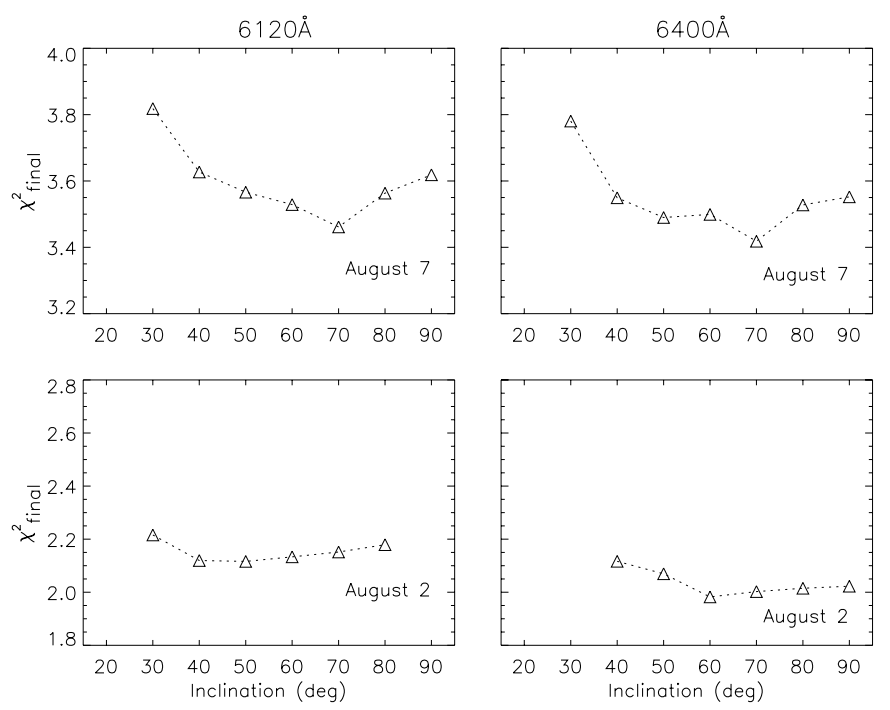

Fig. 8. Quality of line profile fits $\chi_{\text {final }}^{2}$ achieved by CLDI for different datasets of BO Mic as a function of the adopted inclination of the reconstruction star. The wavelength ranges and observation dates corresponding to each graph are annotated. 


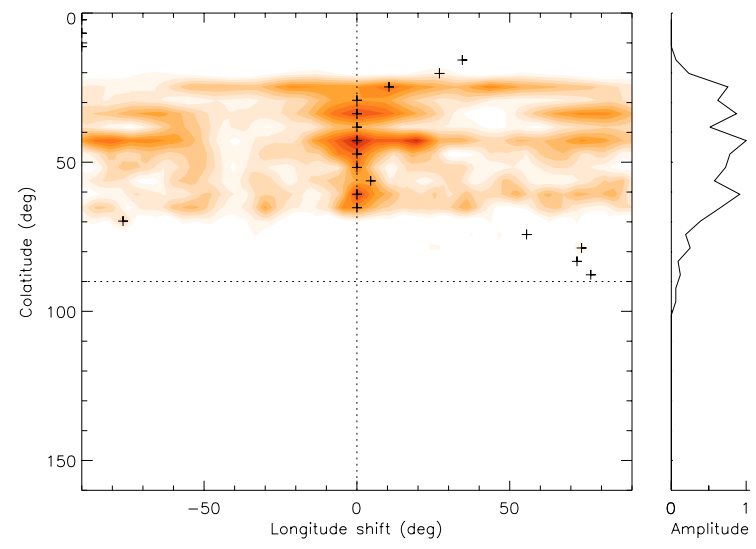

Fig. 9. Quantifying the Doppler image resolution: cross-correlation of different-wavelength Doppler images reconstructed from the same input spectra (2004 August 2, i.e. the upper maps of Fig. 6). The left panel shows the cross-correlation of corresponding latitude strips of the compared maps; darker shades represent larger values. The relative maximum on each colatitude $\left(=90^{\circ}-\right.$ latitude $)$ is marked by a plus, the values of these maxima are plotted in the right panel. See text for details.

$\mathrm{A}_{1}$ : a smaller spot (group) centered slightly below $30^{\circ}$ latitude and at about $290^{\circ}$ longitude;

B: an extended area sparsely covered with spots, centered close to $0^{\circ}$ longitude and $30^{\circ}$ latitude; its shape is reminiscent of a " $\lambda$ ";

$\mathrm{C}_{0}$ : a spot group extending from about $50^{\circ}$ to $120^{\circ}$ in longitude and from close to the equator to about $60^{\circ}$ in latitude. In the August 2 images this group is far more pronounced, showing a separation in two distinct, apparently quite coherent groups;

$\mathrm{C}_{\mathrm{s}}$ : the small coherent southern spot only visible in the August 7 images at about $110^{\circ}$ longitude and $-25^{\circ}$ latitude. It may be mirrored to the northern hemisphere in the August 2 images, appearing there as part of group $C_{0}$.

The following evolution of the spot pattern appears to have taken place during the about $13\left(\frac{5 \text { days }}{0.38 \text { days }}\right)$ rotations between the August 2 and August 7 images: group $A_{0}$ undergoes rather weak changes but is shifted in longitude by roughly $30^{\circ}$. Equivalently, $A_{0}$ could be said to stay in place and fade at lower longitudes while growing at its high longitude side. Group $A_{1}$ appears to stay in place, getting connected to $A_{0}$. Group $B$ remains largely unchanged, preserving its $\lambda$-shape and growing a weak "annex" to the north west at about $320^{\circ}$ longitude and $45^{\circ}$ latitude. Finally, most of group $\mathrm{C}_{0}$ disappears with possibly one component reappearing in the spot $\mathrm{C}_{\mathrm{s}}$.

The apparent shift of group $A_{0}$ towards increasing longitudes (i.e. earlier rotation phases, keeping in mind that rotation takes place with decreasing sub-observer longitude), combined with the pronounced weakening of group $\mathrm{C}_{0}$ at lower longitudes (i.e. later rotation phases) leads to a "darkening" at earlier phases and a "brightening" at later ones. As a result, the lightcurve minimum is shifted to earlier phases when going from August 2 to August 7; this can be seen in the synthetic lightcurves computed from the Doppler images, shown in Fig. 10.

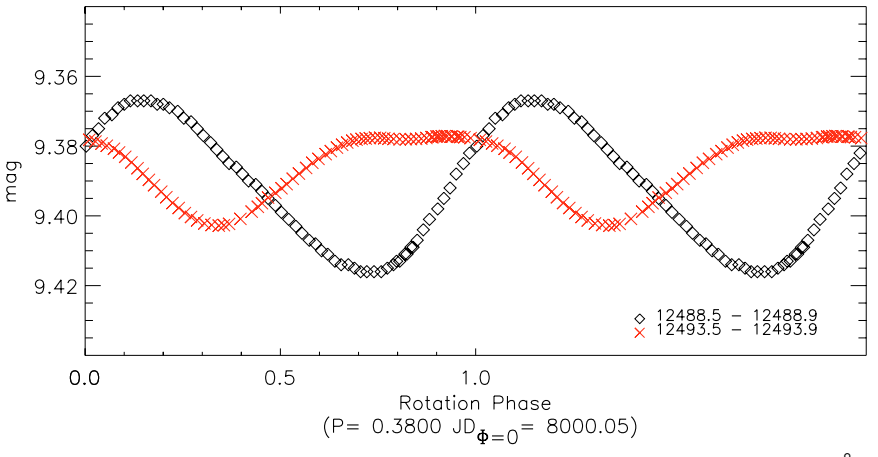

Fig. 10. Lightcurves computed from Doppler images of the "6400 $\AA$ " wavelength region. The symbols represent the August $2(\diamond)$ and August $7(\times)$ reconstructions, respectively. The lightcurves are computed from the corresponding surfaces shown in Fig. 6. The annotation dates are JD-2 440000.

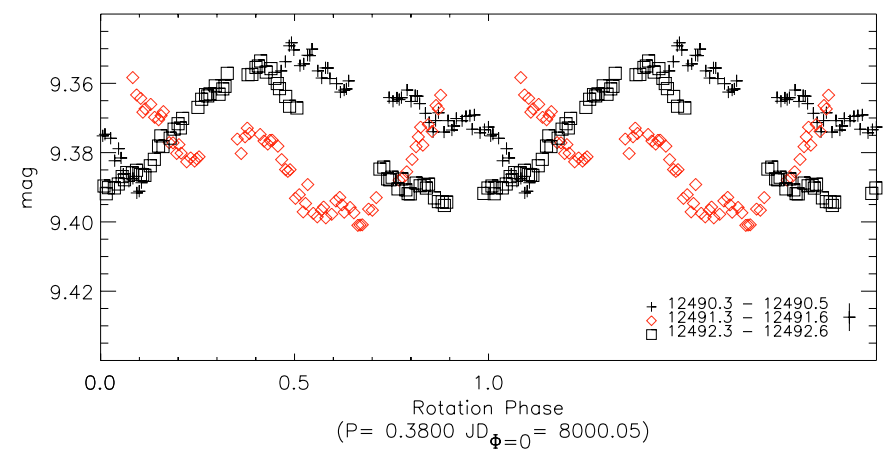

Fig. 11. Lightcurve of BO Mic (2002 August 3-5), folded with a period of 0.380 days. Note that this folding period does not merge any portion of the data observed during different nights into a unique lightcurve. Annotation dates are JD-2 440 000: + = August 3, $\diamond=$ August 4 and $\square=$ August 5 .

The available photometric lightcurve (2004 August 3-5) could not be observed strictly parallel to the spectroscopic observations used for the Doppler imaging; even though it was observed on three consecutive nights, it contains unobserved gaps each lasting about 1.5 rotations due to the short rotation period of BO Mic. However, the available lightcurve indicates that spot reconfigurations comparable to those seen in the Doppler images have occurred on time scales as short as about two rotations (namely between two consecutive nights). Due to the merely disk-integrated information about the spot pattern contained in the lightcurve, these spot reconfigurations cannot be further specified.

The inferred fast changes of some of BO Mic's spots during the August 2002 observations on the one hand, and the snapshot-like information of the Doppler images (as well as the lightcurves) on the other, make definite statements about the spot lifetimes difficult. However, some inferences can be made:

(a) several spot groups of irregular shape, typically extending $10-20^{\circ}$ on the surface, located from equatorial up to about $60^{\circ}$ latitudes, reappear quite well-preserved after 13 rotations;

(b) one quite coherent spot group of about $30^{\circ} \times 30^{\circ}$ surface extension has substantially weakened after 13 rotations; 
(c) the only large spot group, quite coherent but of irregular shape, extends more than $100^{\circ}$ in longitude and about $50^{\circ}$ in latitude. It reappears after 13 rotations with a comparable surface extension but significantly shifted and transformed on scales of up to $30^{\circ}$.

A detailed comparison between our Doppler images of BO Mic and those of Barnes et al. (2001) is difficult. The reliability of small-scale features in their images is presumably low, because of partly low SNR and partly rather inhomogeneous phase coverage of their input spectra. However, the large-scale distribution of spots does show similarities. Barnes et al.'s 1998 images of BO Mic, as well as our 2002 images presented here, both show spots over a wide range of latitudes including regions close to the equator. Both sets of images show one large spot group extending up to or very close to the poles, but neither the 1998 images nor the 2002 images exhibit a polar spot. Our 2002 images clearly show that the hemisphere opposite to the mentioned large spot group is considerably less densely spotted; this is supported by the modulation of the observed lightcurves (Fig. 11). Two differently spotted hemispheres also exist in some of Barnes et al.'s 1998 images. However, we think that due to the partly unfavourable phase sampling of the 1998 observations of BO Mic, no definite statement can be made about this.

It must be kept in mind that all identified spot groups found in the Doppler images may comprise groups truly located on both stellar hemispheres, incorrectly reconstructed only on the northern hemisphere by the Doppler imaging.

\subsection{Differential rotation?}

The intermediate to high latitude spot group $A_{0}$ is apparently shifted to larger longitudes (i.e. against the direction of rotation) by about $30^{\circ}$, while features closer to the equator (including group $A_{1}$ ) roughly remain stationary; this result of a visual inspection is confirmed by the surface cross-correlation shown in Fig. 12 (see Sect. 5.4 for an explanation of the crosscorrelation procedure). However, the surface shear visible in the cross-correlation map is not at all smoothly increasing from the equator to the poles as would be the case for a differential rotation law comparable to the solar case (Eq. (1)). Instead, following the discussion of the preceeding section, we suggest that the apparent surface shear is predominantly due to the intrinsic evolution of the individual spot groups, i.e. their appearance and decay.

As a result our 2004 Doppler images of BO Mic only allow us to estimate an upper limit of the differential rotation strength which could be veiled by the intrinsic spot evolution: Inspecting Fig. 12 yields a pole-equator shear estimate of $\Delta \varphi \approx-20 \pm 10^{\circ}$. The estimated scatter of $\pm 10^{\circ}$ corresponds well with the resolution estimate of our Doppler images made in Sect. 5.4. This translates into a "relative strength" (cf. Eq. (2)) of the differential rotation of

$|\alpha|<\left|\frac{-20 \pm 10 \mathrm{deg}}{13.2 \cdot 360 \mathrm{deg}}\right|=0.004 \pm 0.002$

i.e. at most 50 times smaller than the solar value.

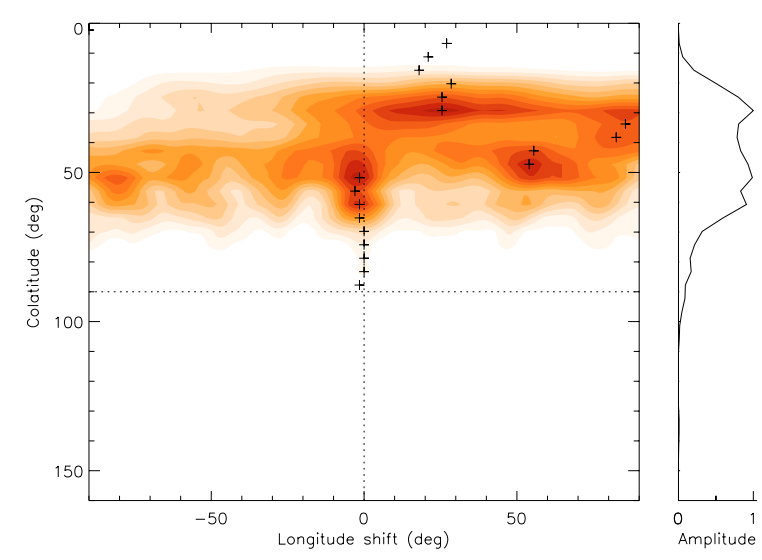

Fig. 12. Cross-correlation of the August 2 and August 7 Doppler images of BO Mic, reconstructed from the "6400 ̊" wavelength region (right column maps of Fig. 6). The compared images have been smoothed by a two-dimensional boxcar extending $10^{\circ} \times 10^{\circ}$ to account for their surface resolution estimated above.

\section{Summary and conclusions}

We have acquired a time series of high-resolution spectra of BO Mic which is of outstanding quality regarding its low noise level and its dense and even sampling of rotation phases. BO Mic shows an exceptionally short rotation period which makes it a very interesting object for studying the spot evolution and possibly the differential rotation of ultrafast rotators (UFR).

Our spectra allow the reconstruction of two Doppler images of BO Mic, seperated by about 13 stellar rotations. Each of these images is based on spectra observed during a single stellar rotation. This minimizes the influence of intrinsic spot evolution on the images, which must be considered a possibility even during a single rotation.

We have applied our spectrum deconvolution algorithm sLSD to extract line profiles from two separate wavelength ranges of the spectra. The Doppler images reconstructed from the resulting two independent input datasets agree on scales down to $10^{\circ} \times 10^{\circ}$ on the stellar surface, excluding latitudes near the pole and the equator. In this way we estimate the surface resolution of our images to about $10^{\circ}$.

While the large-scale spot pattern has survived the 13 rotations covered by our images, several reconfigurations of spots have definitely taken place during that time span. These reconfigurations extend up to about $30^{\circ} \times 30^{\circ}$ on the surface.

While our Doppler images show the timescale of these spot reconfigurations to be shorter than 13 rotations of $\mathrm{BO}$ Mic, our nearly simultaneous photometric observations suggest that similar reconfigurations have even taken place between consecutive observing nights, i.e. during about 2.5 stellar rotations.

Currently, for stars other than the Sun only very few observations of spot lifetimes on timescales of a few stellar rotations exist. By far the best-studied object concerning shortterm spot evolution is the Sun. Compared to the solar case, "fast" changes of the spot pattern are not surprising: pratically no sunspot group survives more than two solar rotations and definitely not without major reconfigurations. 


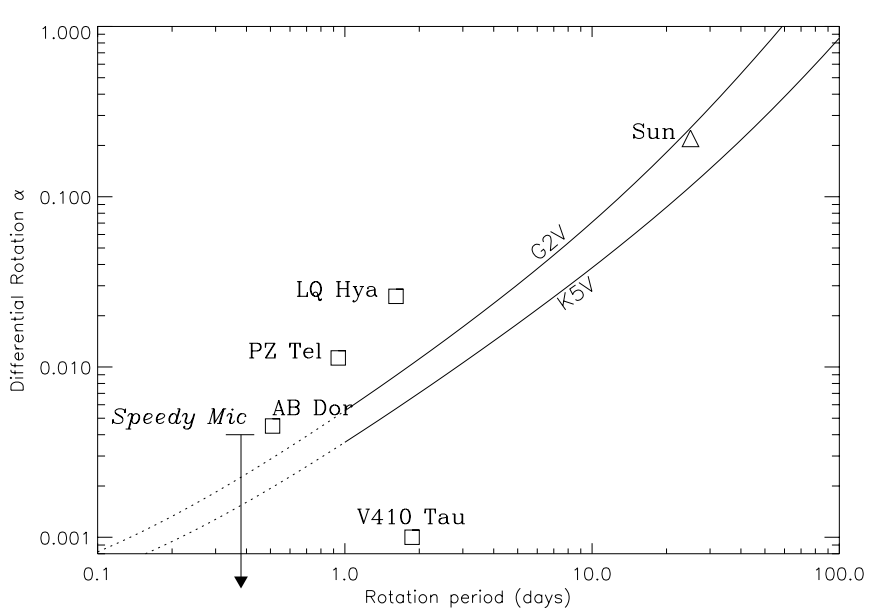

Fig. 13. Theory and measurements of differential rotation of (apparently) single dwarf stars. The curves are adapted from Kitchatinov \& Rüdiger (1999) and extrapolated below a period of one day. Squares mark measurements based on comparisons of Doppler images (Rice \& Strassmeier 1996; Barnes et al. 2000; Donati et al. 1999, 2003) or the related method of "direct spot tracking" (Collier Cameron et al. 2002). The upper limit determined for BO Mic (Speedy Mic) in this work is rendered by the arrow's top. The arrowhead drawn outside the plot illustrates the possibility of anti-solar differential rotation (i.e. the equator rotating slower than the poles in terms of $\Omega$ ) which is not excluded by our observations.

The observed spectral line profiles of BO Mic contain remarkably rich information which agrees between the different wavelength ranges. This fact and the resulting quality of the Doppler images make our observations highly successful. However, one of our major aims could not be achieved, namely the observation of differential rotation on BO Mic and the determination of its strength. Given the large-scale stabilty of the spot pattern deduced from our Doppler images, the differential rotation can safely be said to be weak compared to the Sun. Unfortunately, further determination of the differential rotation strength is massively impaired by the above-discussed intrinsic evolution of the observed spots.

Our determined upper limit of $|\alpha|<0.004 \pm 0.002$ for the strength of the differential rotation agrees well with a previously determined value by Barnes et al. (2001). Combined with measurements of differential rotation on other fast rotating dwarf stars, shown in Fig. 13, it confirms the observed pronounced decline of differential rotation strength with decreasing rotation periods. As Fig. 13 also illustrates, this is well in accord with the rotational models of Kitchatinov \& Rüdiger (1999), so far supporting the mean-field-based modelling approach for the convection zones of solar-like stars.

Acknowledgements. U.W. acknowledges financial support from Deutsche Forschungsgemeinschaft, DFG - SCHM 1032/9-1. We thank D. Kilkenny for making the photometric observations at SAAO possible and G. Cutispoto for kindly supplying photometric data of Speedy Mic. Finally, we thank our referee J. Barnes for his careful reading of the manuscript and his concise suggestions which helped to improve it.

\section{References}

Anders, G. J., Jeffries, R. D., Kellet, B. J., \& Coates, D. W. 1993, MNRAS, 265, 941

Barnes, J. R., Collier Cameron, A., Unruh, Y. C., Donati, J.-F., \& Hussain, G. A. J. 1998, MNRAS, 299, 904

Barnes, J. R., Collier Cameron, A., James, D. J., \& Donati, J.-F. 2000, MNRAS, 314, 162

Barnes, J. R., Collier Cameron, A., James, D. J., \& Donati, J.-F. 2001, MNRAS, 324, 231

Barnes, J. R., 2003, MNRAS, 348, 1295

Beck, J. G. 1999, Sol. Phys., 191, 47

Bromage, G. E., Jeffries, R. D., Innis, J. L., et al. 1992, ASP Conf. Ser., 26, 80

Collier Cameron, A., Donati, J. F., \& Semel, M. 2001, MNRAS, 330, 699

Cox, A. ed. 2000, Allen's Astrophysical Quantities (New York: Springer)

Cutispoto, G., Kürster, M., Pagano, I., \& Rodono, M. 1997, Information Bulletin on Variable Stars, 4419, 1

Donati J.-F., \& Collier Cameron, A. 1997, MNRAS, 291, 1

Donati J.-F., Semel, M., Carter, B. D., Rees, D. E., \& Collier Cameron, A. 1997, MNRAS, 291, 658

Donati, J.-F., Collier Cameron, A., Hussain, G. A. J., \& Semel, M. 1999, MNRAS, 302, 437

Donati, J.-F., Collier Cameron, A., \& Petit, P. 2003, MNRAS, 345, 1187

Gizon, L., Duvall Jr., T. L., \& Schou, J. 2003, Nature, 421, 43

Gray, D. F. 1992, The observation and analysis of stellar photospheres (Cambridge: Cambridge Univ. Press)

Hauschildt, P., \& Allard, F., ApJ, 512, 377

Horne, K. 1986, PASP 98, 1220

Hussain, G. A. J., 2002, AN, 323, 349

Jankov, S., \& Foing, B. H. 1992, A\&A, 256, 533

Kilkenny, D., Balona, L. A., Carter, D. B., Ellis, D. T., \& Woodhouse, G. F. W. 1988, Mon. Notes Astron. Soc. S. Afr., 47, 69

Kilkenny, D., van Wyk, F., Roberts, G., Marang, F., \& Cooper, D., 1998, MNRAS, 294, 93

Kitchatinov, L. L., \& Rüdiger, G. 1999, A\&A, 344, 911

Kürster, M. 1993, A\&A, 274, 851

Lucy, L. B. 1994, Rev. Mod. Astron., 7, 31

Menzies, J. W., Cousins, A. W. J., Banfield, R. M., \& Laing, J. D. 1989, SAAOC, 13, 1

Montes, D., Lopez-Santiago, J., Galvez, M. C., et al. 2001, MNRAS, 328, 45

Press, W. H., Flannery, B. P., Teukolsky, S. A., \& Vetterling, W. T., Numerical recipes in C (2nd ed.) (Cambridge: Cambridge Univ. Press)

Reiners, A., \& Schmitt, J. H. M. M. 2002, A\&A, 384, 155

Reiners, A., \& Schmitt, J. H. M. M. 2003, A\&A, 398, 647

Rice, J.B., \& Strassmeier, K. G. 1996, A\&A, 316, 164

Rice, J.B., \& Strassmeier, K. G. 2001, A\&A, 377, 264

Saar, S. H., Peterchef, A., O’Neal, D., \& Neff, J. E. 2001, ASP Conf. Ser. 323, 1057

Siess, L., Dufour, E., \& Forestini, M. 2000, A\&A, 358, 593

Soderblom, D. R., Jones, B. F., Balachandran, S., et al. 1993, ApJS, 85,315

Tonry, J., \& Davis, M. 1979, AJ, 84, 1511

Vogt, S. S., Penrod, G. D., \& Hatzes, A. P. 1987, ApJ, 321, 496

Vogt, S. S., Hatzes, A. P., \& Misch, A. A. 1999, ApJS, 121, 547

Weber, M., \& Strassmeier, K. G. 2001, A\&A, 373, 974

Wöhl, H. 2002, AN, 323, 329

Wolter, U. 2004, Ph.D. Thesis, Hamburg 